\title{
Suppression of MOG- and PLP-Induced Experimental Autoimmune Encephalomyelitis Using a Novel Multivalent Bifunctional Peptide Inhibitor
}

\author{
Ahmed H. Badawi ${ }^{1}$ and Teruna J. Siahaan ${ }^{1,2}$ \\ ${ }^{1}$ Department of Pharmaceutical Chemistry, University of Kansas, Lawrence, KS 66047
}

\begin{abstract}
Previously, bifunctional peptide inhibitors (BPI) with a single antigenic peptide have been shown to suppress experimental autoimmune encephalomyelitis (EAE) in an antigen-specific manner. In

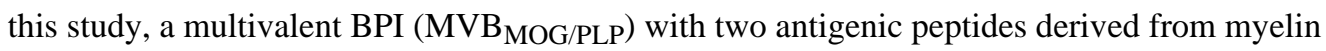
oligodendrocyte glycoprotein $\left(\mathrm{MOG}_{38-50}\right)$ and myelin proteolipid protein $\left(\mathrm{PLP}_{139-151}\right)$ was evaluated in suppressing $\mathrm{MOG}_{38-50^{-}}$and $\mathrm{PLP}_{139-151^{-} \text {-induced EAE. MVB }} \mathrm{MOG}_{\mathrm{PLP}}$ significantly suppressed both models of EAE even when there was some evidence of epitope spreading in the

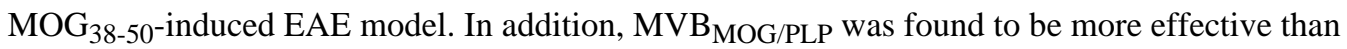
PLP-BPI and MOG-BPI in suppressing $\mathrm{MOG}_{38-50}$-induced EAE. Thus, the development of MVB molecules with broader antigenic targets can lead to suppression of epitope spreading in EAE.
\end{abstract}

\section{Keywords}

Experimental autoimmune encephalomyelitis; Bifunctional peptide inhibitor; Antigen-presenting cell; T cell; Epitope spreading

\section{Introduction}

Multiple sclerosis (MS) is an immune-mediated neurodegenerative disease of the central nervous system (CNS). The pathogenesis of MS has not been fully elucidated; however, it is categorized as a CD4 ${ }^{+} \mathrm{T}$ cell-mediated autoimmune disease (Bielekova et al., 1999, Zhang et al., 1994). It is thought that there is a breakdown in the recognition of self versus non-self antigens, and that the immune system starts recognizing protein components of the myelin sheath as antigens. Major immunodominant proteins of the myelin sheath are myelin proteolipid protein (PLP), myelin oligodendrocyte glycoprotein (MOG), and myelin basic protein (MBP). During disease, T cells can recognize epitopes of these proteins and initiate an inflammatory immune response toward them, leading to tissue damage. The debris from

\footnotetext{
(C) 2013 Elsevier B.V. All rights reserved.

${ }^{2}$ Address correspondence to: Teruna J. Siahaan, Department of Pharmaceutical Chemistry, The University of Kansas, 2095 Constant Avenue, Lawrence, Kansas 66047, Phone: 785-864-7327, Fax: 785-864-5736, siahaan@ ku.edu.

Publisher's Disclaimer: This is a PDF file of an unedited manuscript that has been accepted for publication. As a service to our customers we are providing this early version of the manuscript. The manuscript will undergo copyediting, typesetting, and review of the resulting proof before it is published in its final citable form. Please note that during the production process errors may be discovered which could affect the content, and all legal disclaimers that apply to the journal pertain.
} 
the broken down tissue leads to epitope spreading, thus resulting in new tissue components becoming antigenic (Vanderlugt and Miller, 1996).

An animal model known as experimental autoimmune encephalomyelitis (EAE) is often used to study MS. This model mimics some of the pathological features of MS such as CNS inflammation, lesion formation, blood-brain barrier (BBB) breakdown, and the presence of myelin-specific CD4 ${ }^{+} \mathrm{T}$ cells (Lassmann, 2001, Voskuhl et al., 1993). In addition, the animal model can be used to study the epitope spreading process (Tuohy and Kinkel, 2000). Unlike MS, however, EAE has an initiating antigen that can be controlled by injection of the encephalotogenic peptide in the presence of complete Freund's adjuvant (CFA). This is a powerful tool because therapies can be developed to specifically suppress the immune response to these antigens. Antigen-specific immunotherapy has become widely investigated recently with the aim of inducing tolerance to specific antigens; therefore, it attenuates the inflammatory response. Previously in our laboratory, bifunctional peptide inhibitors (BPI) composed of antigenic peptides conjugated to adhesion peptides have been developed and have successfully suppressed EAE (Kobayashi et al., 2007, Kobayashi et al., 2008, Ridwan et al., 2010, Zhao et al., 2010, Manikwar et al., 2011). BPI molecules contain a specific antigen (i.e., PLP) and have been shown to suppress EAE induced by a specific antigen (i.e., PLP). Therefore, they will not be useful for suppressing EAE generated by a different antigen (i.e., MOG or MBP). In addition, antigen-specific modulation may not solve the problem of epitope spreading when the disease is in its late stage. Therefore, a new kind of BPI molecule known as multivalent BPI (MVB) has been designed with more than one antigen. The goal is that the MVB molecule will modulate the immune response to suppress the disease regardless of the inciting antigen, thus solving the problem of epitope spreading and making this strategy more applicable for translation into a MS therapy.

In $\mathrm{EAE}$ and $\mathrm{MS}$, the activation of inflammatory $\mathrm{CD}^{+} \mathrm{T}$ cells is mediated by two signalsthat are delivered from antigen-presenting cells (APC) to T cells (Grakoui et al., 1999, Tseng and Dustin, 2002). The first signal (Signal 1) is the antigen presentation by the major histocompatibility complex class-II (MHC-II) molecule, which is recognized by the T-cell receptor (TCR). The second signal (Signal 2) is made up of costimulatory and adhesion molecules on both APC and T cells. After the molecular pair interactions form both signals, signal translocation occurs to form the immunological synapse that leads to activation of a sub-population of antigen-specific T cells (Lee et al., 2002, van der Merwe, 2002). The hypothesis is that BPI molecules are designed to simultaneously target Signal 1 and adhesion molecules on the surface of APC to hinder the formation of the immunological synapse, which will prevent the activation of the inflammatory $\mathrm{T}$ cells that specifically recognize the antigenic portion of the BPI molecule. MVB molecules are composed of more than one antigenic peptide, and can therefore bind to different MHC-II molecules on the same or different APC. Thus, the inflammatory response toward more than one antigen is prevented.

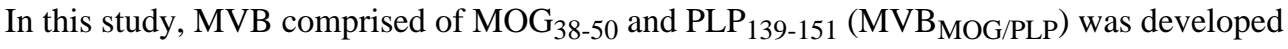
and evaluated for suppressing two different antigen-induced EAE models. As controls, MOG-BPI and PLP-BPI were evaluated for cross-reactivity in MOG-induced and PLPinduced EAE. In this case, the efficacy of MOG-BPI was evaluated in PLP-induced EAE, 
and the efficacy of PLP-BPI was evaluated in MOG-induced EAE. Finally, some mechanistic aspects of MVB were elucidated by determining the cytokines produced by splenocytes of the EAE animals upon treatment.

\section{Materials and Methods}

\subsection{Mice}

All protocols for experiments involving SJL/J $\left(\mathrm{H}-2^{\mathrm{S}}\right)$ (Charles River, Wilmington, MA) and C57BL/6 (Jackson Laboratory, Bar Harbor, ME) were approved by the University's Institutional Animal Care and Use Committee. The mice were housed under specific pathogen-free conditions at a facility at the University of Kansas, which is approved by the Association for Assessment and Accreditation of Laboratory Animal Care.

\subsection{Peptide Synthesis}

Peptides used in this study are listed in Table 1. 9-Fluorenylmethyloxy-carbonyl-protected amino acid chemistry was used to synthesize all peptides, utilizing an appropriate PEG-PSTM resin (Applied Biosystems, Foster City, CA) in an automated peptide synthesis system (Pioneer ${ }^{\mathrm{TM}}$ :PerSeptive Biosystems, Framingham, MA). The peptides were cleaved from the resin, and removal of the protecting groups from the side-chain was accomplished with $90 \%$ TFA with $10 \%$ scavenger reagents (1,2-ethane dithiol (3\%), anisole (2\%), and thioanisole $(5 \%))$. The crude products were purified by reversed-phase HPLC using a semi-preparative $\mathrm{C} 18$ column with a gradient of solvent $\mathrm{A}\left(95 \% / 5 \%=\mathrm{H}_{2} \mathrm{O}(0.1 \%\right.$ TFA $) /$ acetonitrile $)$ and solvent B (100\% acetonitrile). Analytical HPLC with a C18 column was used to determine the purity of each peptide. The identity of each synthesized peptide was confirmed by electrospray ionization mass spectrometry.

\subsection{Induction of EAE and Clinical Evaluation}

For the PLP-induced EAE, SJL/J female mice (5-7 weeks old) were immunized subcutaneously (s.c.) with $200 \mu \mathrm{g}$ of PLP139-151 peptide in a $0.2 \mathrm{ml}$ emulsion comprised of equal volumes of phosphate-buffered saline (PBS) solution and CFA containing killed mycobacterium tuberculosis strain H37RA at a final concentration of $4 \mathrm{mg} / \mathrm{ml}$ (Difco, Detroit, MI). The PLP/CFA emulsion was administered to regions above the shoulder and the flanks (total of 4 sites; $50 \mu \mathrm{l}$ at each injection site). In addition, $200 \mathrm{ng}$ of pertussis toxin (List Biological Laboratories, Campbell, CA) was injected intraperitoneally (i.p.) on the day of immunization (day 0 ) and $48 \mathrm{~h}$ post-immunization.

For the MOG-induced EAE, C57BL/6 mice (4-6 weeks old) were immunized in a fashion similar to that mentioned above, except that $200 \mu \mathrm{g}$ of $\mathrm{MOG}_{38-50}$ peptide was used and 400 $\mathrm{ng} /$ mouse/injection of pertussis toxin was administered on days 0 and 2 . The clinical scores that reflect the disease progression were determined by the same observer in a blinded fashion using a scale ranging from 0 to 5 as follows: 0 - no clinical symptoms, 1 - limp tail or waddling gait with tail tonicity; 2 - waddling gait with limp tail (ataxia); 2.5 - ataxia with partial paralysis of one limb; 3 - full paralysis of one limb; 3.5 - full paralysis of one limb with partial paralysis of the second limb; 4 - full paralysis of two limbs; 4.5 - full paralysis 
of two limbs with partial paralysis of forelimbs; 5 - moribund or dead. Body weight was also measured daily.

\subsection{In vivo Peptide Treatments}

Study I - Cross reactivity of MOG-BPI and PLP-BPI-This study was performed to study the in vivo cross-reactivity of MOG-BPI and PLP-BPI in suppressing EAE. This was achieved upon induction of the disease with one antigen followed by treating the animals with a BPI molecule containing another antigen. As positive controls, the in vivo efficacies of MOG-BPI and PLP-BPI were evaluated to suppress MOG- and PLP-induced EAE, respectively. Induction of the disease was performed on day 0 as described in section 2.3. In the MOG-induced EAE, each mouse received s.c. injections of PLP-BPI and MOG-BPI at a concentration of $100 \mathrm{nmol} / 100 \mu \mathrm{l} /$ injection (in PBS) on days 4, 7, and 10. The efficacies of both PLP-BPI and MOG-BPI were compared to that of the vehicle (PBS). In the PLPinduced EAE, MOG-BPI was administered s.c. at a concentration of $100 \mathrm{nmol} / 100 \mu \mathrm{l} /$ injection (in PBS) on days 4, 7, and 10. The efficacy of each peptide was evaluated by monitoring the clinical score and the change in body weight over a period of 25 days.

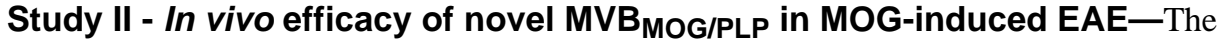
purpose of this study was to evaluate the in vivo efficacy of the novel MVB $\mathrm{MOG}_{\mathrm{PLP}}$ in suppressing MOG-induced EAE. Mice were immunized with MOG/CFA on day 0 as described in section 2.3. The first group of mice received three s.c. injections of $\mathrm{MVB}_{\mathrm{MOG} / \mathrm{PLP}}$ at a concentration of $100 \mathrm{nmol} / 100 \mu \mathrm{l}$ (in PBS) on days 4, 7, and 10, and its efficacy was compared to those of the vehicle (100 $\mu \mathrm{l}$ PBS) and positive controls, MOG $(100 \mathrm{nmol} / 100 \mu \mathrm{l})$ and MOG-BPI $(100 \mathrm{nmol} / 100 \mu \mathrm{l})$. The negative (PBS) control and the positive control were each injected three times on days 4, 7, and 10. The efficacy of each treatment was evaluated using the clinical score and the change in body weight over a period of 25 days.

Study III - In vivo efficacy of novel MVB ${ }_{M O G / P L P}$ in PLP-induced EAE-The efficacy of MVB $\mathrm{BOG}_{\mathrm{PLP}}$ was also evaluated in PLP-induced EAE. All mice were immunized with PLP/CFA on day 0 as described in section 2.3. One group of mice received three s.c. injections of MVB $\mathrm{MOG}_{\mathrm{PLP}}$ at a concentration of $100 \mathrm{nmol} / 100 \mu \mathrm{l}$ on days 4,7 , and 10; another group received $100 \mu \mathrm{l}$ of vehicle (PBS) s.c. on the same days. The efficacy of the peptide was evaluated by monitoring the clinical score and change in body weight over a period of 25 days.

\subsection{In vitro Inflammatory Cytokine Production Assay}

In vitro cytokine assays were performed following a protocol similar to that reported previously (Youssef et al., 2002). Cytokines produced from MOG-induced C57BL/6 mice treated with MOG-BPI and $\mathrm{MVB}_{\mathrm{M}} \mathrm{og} / \mathrm{plp}$ were measured and compared to that from PBStreated mice. EAE was induced by injection of MOG/CFA and pertussis toxin as described in section 2.3, and mice were treated with PBS $(100 \mu \mathrm{l})$, MOG-BPI $(100 \mathrm{nmol} / 100 \mu \mathrm{l} /$ injection), or MVBmog/plp (100 nmol/100 $\mu \mathrm{l} /$ injection) on days 4, 7, and 10. Spleens were isolated from three mice from each group on day 30. Single cell suspensions of splenocytes were harvested by gently mashing the spleen through a cell strainer using the rubber end of a 
1-ml syringe in a petri dish containing serum-free RPMI-1640 supplemented with $10 \%$ fetal bovine serum, $100 \mathrm{U}$ penicillin/100 $\mu$ g streptomycin, $2 \mathrm{mM} \mathrm{L-glutamine,} \mathrm{and} 50 \mu \mathrm{M} 2$ mercaptoethanol. Red blood cells were lysed using ACK lysis buffer (Invitrogen). The remaining splenocytes were then washed three times with serum-free RPMI-160 medium (Cellgro). The cells were then primed with PLP $(20 \mu \mathrm{M})$ in a 24 -well plate $\left(5 \times 10^{6}\right.$ cells/ well). Supernatants of cell cultures were collected for cytokine detection 72 hours later and stored in a $-80^{\circ} \mathrm{C}$ freezer until analysis. Secreted IL- 6 and IFN- $\gamma$ were measured by quantitative ELISA-based Q-Plex ${ }^{\mathrm{TM}}$ assay (Quansys Biosciences, Logan, UT).

\subsection{Splenocyte Proliferation Assay}

A proliferation assay was conducted in SJL/J mice in order to evaluate the cross-reactivity between MOG and PLP. This was accomplished by isolating splenocytes from three PLPinduced EAE mice per group on day 30 as described in section 2.5. Splenocytes were isolated from four different groups. One group consisted of mice that had no EAE induced. The next three groups were mice treated with PBS $(100 \mu \mathrm{l})$, PLP-BPI $(100 \mathrm{nmol} / 100 \mu \mathrm{l} /$ injection), or $\mathrm{MVB}_{\mathrm{MOG} / \mathrm{PLP}}(100 \mathrm{nmol} / 100 \mu \mathrm{l} / \mathrm{injection})$ on days 4,7 , and 10 . The cells were cultured and stimulated with PLP $(2 \mu \mathrm{M})$, MOG $(2 \mu \mathrm{M})$ or concanavalin A (positive control). Cells were cultured in a 96-well plate $\left(2 \times 10^{5}\right.$ cells/100 $\mu 1 /$ well $)$ for 72 hours. Cultures were then pulsed overnight with $1 \mu \mathrm{Ci}$ of $\left[{ }^{3} \mathrm{H}\right]$ thymidine per well. Cells were harvested onto glass fiber filters using the FilterMate Harvester (PerkinElmer), and the unincorporated $\left[{ }^{3} \mathrm{H}\right]$ thymidine was removed by multiple washes according to the procedure recommended by the manufacturers. The incorporated radioactivity was then counted using a $\beta$-scintillation counter (Microbeta Trilux, PerkinElmer).

\subsection{Statistical Analysis}

Statistical analysis was done using one-way analysis of variance followed by Fisher's least significance difference to compare the different parameters, including in vitro cytokine production, splenocyte proliferation, and values from days 10-25 (unless otherwise stated) for EAE clinical scores and changes in body weight. All statistical analyses were performed using StatView software (SAS Institute, Inc., Cary, NC). A p-value of less than 0.05 was used as the criterion for statistical significance.

\section{Results}

\subsection{Study I - Cross Reactivity of MOG-BPI and PLP-BPI}

MOG-BPI's efficacy in suppressing MOG-induced EAE was evaluated for the first time. To test whether there is cross-reactivity between the different antigens, PLP-BPI was tested for suppressing MOG-induced EAE. The PBS-treated mice developed very severe EAE with a high average clinical score of 4.0 and $22.15 \%$ loss in body weight compared to the day of onset of disease. The best suppression was observed in the MOG-BPI-treated mice, with a significant difference in clinical scores and changes in body weight on days 15-25 compared to those of the PLP-BPI-treated group ( $p<0.01$ for clinical score and $p<0.0001$ for change in body weight) and the PBS-treated group ( $p<0.0001$ for clinical score and change in body weight). PLP-BPI significantly suppressed MOG-induced EAE on days 15-25 compared to 
PBS as indicated by clinical score $(p<0.01)$ and change in body weight $(p<0.001)$ (Fig. $1 \mathrm{~A}$ and Fig 1B).

For the second part of this study, the efficacy of MOG-BPI was evaluated in suppressing PLP-induced EAE. The PBS-treated mice exhibited signs of EAE with a maximal clinical score of 1.67 and a maximal loss in body weight of $13.2 \%$. The MOG-BPI-treated mice showed similar signs of EAE with a maximal clinical score of 1.5 and a maximal loss in body weight of $11.69 \%$. There were no significant differences in clinical score $(p>0.05)$ between the PBS-treated mice and the MOG-BPI-treated mice (Fig. 1C and Fig. 1D).

\subsection{Study II - In vivo Efficacy of Novel MVB ${ }_{M O G / P L P}$ in MOG-Induced EAE}

For this study, the efficacy of the novel MVB $\mathrm{MOG}_{\mathrm{MOLP}}$ peptide was tested in suppressing MOG-induced EAE and was compared to that in PBS-, MOG-, and MOG-BPI-treated mice. As expected, PBS-treated mice developed severe EAE with a maximal clinical score of 3.58 and a $24.14 \%$ loss in body weight. The greatest suppression of disease was observed in mice treated with the novel MVB ${ }_{M O G / P L P}$. Clinical scores from days 15-25 showed significant suppression in the $\mathrm{MVB}_{\mathrm{MOG} / \mathrm{PLP}}$-treated mice compared to PBS-, MOG-, and MOG-BPItreated mice (Fig. $2 \mathrm{~A}, p<0.0001$ ). The $\mathrm{MVB}_{\mathrm{MOG} / \mathrm{PLP}}$-treated group reached a maximal disease score of only 0.88 and $5.15 \%$ loss in body weight. Similarly, the change in body weight of $\mathrm{MVB}_{\mathrm{MOG} / \mathrm{PLP}}$-treated mice on days 15-25 showed significant differences compared to those of PBS-, MOG-, and MOG-BPI-treated mice (Fig. 2B, $p<0.0001$ ). Treatment with MOG-BPI delayed onset of disease by four days. The clinical scores and loss in body weight indicated that it suppressed disease significantly compared to PBS ( $p<$ 0.0001). Clinical scores indicated that MOG-BPI was significantly more efficacious than MOG peptide ( $p<0.05$, days 15-25); however, there was no significant difference in the change of body weight between MOG-BPI- and MOG-treated mice. Compared to PBStreated mice, the MOG-treated mice showed significant suppression of disease $(p<0.0001)$ with only $10.88 \%$ loss in body weight and a maximum average clinical score of 2.42 .

\subsection{Study III - In vivo Efficacy of Novel MVB ${ }_{M O G / P L P}$ in PLP-Induced EAE}

The purpose of this study was to evaluate whether our novel peptide's efficacy in MOGinduced EAE can be translated to PLP-induced EAE. Therefore, mice were given three s.c. injections of $\mathrm{MVB}_{\mathrm{MOG} / \mathrm{PLP}}$, and its efficacy was compared to that of PBS. According to clinical score data, $\mathrm{MVB}_{\mathrm{MOG} / \mathrm{PLP}}$ suppressed disease significantly $(p<0.0001)$ while the PBS-treated mice reached a maximal score of 1.67 and MVB MOG/PLP-treated mice reached a maximum of 0.58 (Fig. 3A). The changes in body weight correlate exactly with the observed differences in the clinical scores $(p<0.001)$, with PBS-treated mice losing $13.20 \%$ of their body weight and $\mathrm{MVB}_{\mathrm{MOG} / \mathrm{PLP}}$-treated mice losing only a maximum of $4.66 \%$ (Fig. 3B).

\subsection{In vitro Inflammatory Cytokine Production}

Splenocytes from treated mice were isolated on day 30 and their cytokine production was measured. EAE is an inflammatory disease characterized by high proliferation of inflammatory $\mathrm{T}_{\mathrm{H}} 1$ and $\mathrm{T}_{\mathrm{H}} 17$ cells. In vitro cytokine studies can be used to indirectly measure how strong the inflammatory response is in the mouse. The two key cytokine 
markers are IL-6 (Fig. 4A), which is important for $\mathrm{T}_{\mathrm{H}} 1$ and $\mathrm{T}_{\mathrm{H}} 17$ differentiation, and IFN- $\gamma$ (Fig. 4B), which is primarily a $\mathrm{T}_{\mathrm{H}} 1$ cytokine. The production of these pro-inflammatory cytokines was lowest in the MVB $\mathrm{MOG}_{\mathrm{PLP}}$-treated mice and was signficantly different compared to those treated with PBS $(p<0.01)$. MOG-BPI reduced IL-6 production and significantly suppressed IFN- $\gamma(p<0.01)$. MVB ${ }_{\text {MOG/PLP-treated mice had significantly }}$ suppressed production of IL-6 compared to MOG-BPI-treated mice $(p<0.05)$ but only a small difference in IFN- $\gamma$.

\subsection{Splenocyte Proliferation}

A proliferation assay was performed to evaluate the extent of responsiveness of splenocytes from the different treatment groups to in vitro antigen stimulation. In addition, the crossreactivity of the antigens was studied. Splenocytes were isolated from PLP-immunized $\mathrm{SJL} / \mathrm{J}$ mice, and their proliferation was measured in non-immunized mice (no EAE) and

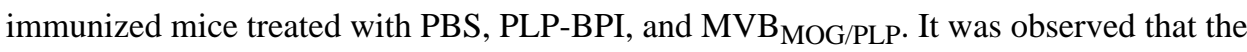
responsiveness of PLP-BPI-treated mice was lower than in the PBS group when the splenocytes were stimulated with PLP $(p<0.05)$. However, MVB ${ }_{\text {MOG/PLP }}$ had the least responsiveness when exposed to PLP. Finally, when the splenocytes were stimulated with MOG, there was no difference in proliferation compared to the splenocytes stimulated with medium. Therefore, the results indicated no cross-reactivity between PLP and MOG in PLPinduced EAE (Fig. 5).

\section{Discussion}

BPI molecules have been developed to target many different antigens responsible for the pathogenesis of autoimmune diseases. PLP-containing BPI molecules have been studied extensively for the suppression of PLP-induced EAE (Kobayashi et al., 2007, Kobayashi et al., 2008, Ridwan et al., 2010, Zhao et al., 2010). GAD-BPI was developed and was successful in suppressing disease in the non-obese diabetic mouse model (Murray et al., 2007). CII-BPI molecules composed of collagen-II antigenic peptides have been shown to suppress collagen-II-induced rheumatoid arthritis in an animal model (unpublished data). Our first goal for this study was to investigate whether BPI molecules composed of other myelin sheath epitopes involved in EAE and MS can be developed to suppress disease. Previously, only PLP-containing BPI molecules were tested. In this study, a novel BPI molecule composed of the MOG antigen (MOG-BPI) was developed and tested in suppressing EAE. While BPI molecules have demonstrated superior efficacy thus far, there is a major limitation to their application. BPI molecules are antigen specific and, therefore, will only suppress the immune response toward other antigens involved in the progression of disease. In reality, MS patients have been shown to respond to many different epitopes (Tuohy et al., 1997, Tuohy et al., 1999a, Tuohy et al., 1999b, Goebels et al., 2000, Muraro et al., 2003), and single antigen-specific immunosuppression could have a disadvantage. Thus, there is a need to expand this technology to target multiple antigens. For the first time, a multivalent BPI molecule has been developed and its efficacy has been tested in two separate EAE models. All in vivo results from the current study are summarized in Table 2.

The antigen specificity of BPI molecules has been previously demonstrated using BPI molecules that contain scrambled and irrelevant peptides. In the irrelevant peptides, PLP 
peptide was replaced with ovalbumin $\left(\mathrm{OVA}_{326-337}\right)$ and an epitope of virus capsid protein (VP2 $74-86$ ) to produce OVA-BPI and VP2-BPI molecules (Kobayashi et al., 2007). OVABPI and VP2-BPI had no or very low efficacy in suppressing EAE, while PLP-BPI had very high efficacy at the same concentration as OVA-BPI and VP2-BPI (Kobayashi et al., 2007). This result suggests that the efficacy of PLP-BPI is due to antigenic specificity of PLP-BPI, and the observed residual efficacy of VP2-BPI is attributed to the cell adhesion peptide LABL peptide in VP2-BPI (Kobayashi et al., 2007). The effect of linking PLP and LABL peptides on efficacy of PLP-BPI was evaluated by comparing the efficacy of PLP-BPI and unconjugated PLP and LABL. PLP-BPI had higher efficacy than a mixture of PLP and LABL. However, the mixture of PLP and LABL had higher efficacy than PLP or LABL peptide alone, suggesting that conjugation of the two peptides is important for efficacy (Kobayashi et al., 2007). PLP-BPIsc with a scrambled PLP sequence also had no efficacy in suppressing EAE, indicating antigenic specificity (Ridwan et al., 2010). Finally, the efficacy comparison between a new molecule called PLP-cIBR and its derivative with a scrambled PLP peptide (i.e., PLPsc-cIBR) showed that PLP-cIBR was very effective in suppressing EAE while PLPsc-cIBR had no efficacy to suppress EAE (Kiptoo et al., 2013). In summary, BPI molecules are significantly effective at suppressing EAE in an antigen-specific manner, and conjugation between the antigenic peptide and the cell adhesion peptides (i.e., LABL or cIBR peptide) has an important role in their action.

The in vivo efficacy and the potential cross-reactivity of $\mathrm{MVB}_{\mathrm{MOG} / \mathrm{PLP}}$, PLP-BPI and MOGBPI were evaluated in both PLP- and MOG-induced EAE. These models represent two different disease states in MS. PLP-induced EAE is an animal model for relapsing-remitting MS (RRMS) and MOG-stimulated EAE is an animal model for primary progressive MS (PPMS). The differences between these two models are the lower severity of the disease in PLP-induced EAE compared to that of MOG-induced EAE and the remitting-relapsing disease course of the PLP-induced EAE. According to our studies, PLP-induced mice show remission around day 25 and relapse around day 45 similar to RRMS while MOG-induced EAE mice do not show any remission of the disease. The results showed that MVB $\mathrm{BOG}_{\mathrm{PLP}}$ suppressed the disease in both models. In PLP-induced mice, PLP-BPI suppressed the disease while MOG-BPI did not, indicating that there was no cross-reactivity between these two BPI molecules in PLP-stimulated EAE. This finding was well correlated with the proliferation assay data showing that there was no response in splenocytes stimulated with MOG. In contrast, a significant suppression of disease was observed with PLP-BPI in MOGstimulated EAE, thus suggesting some cross-reactivity of PLP-BPI in this model.

Interestingly, MVB ${ }_{M O G / P L P}$ suppressed the disease better than MOG-BPI in MOG-induced EAE, suggesting that there is an additive effect of potency when both antigenic peptides (i.e., PLP and MOG) are conjugated in one molecule (i.e., MVB $\mathrm{MOG}_{\mathrm{PLP}}$ ). We propose that the cross-reactivity of PLP-BPI and the better efficacy of MVB ${ }_{M O G / P L P}$ than MOG-BPI in MOG-induced EAE are caused by the ability of these BPI molecules to overcome epitope spreading that includes PLP antigen. Due to the chronic and progressive nature of MOGinduced EAE, the disease progression could cause the immune cells to become sensitive not only to epitopes from MOG but also to PLP and/or MBP (i.e., antigenic spreading). 
A key factor in the progression of MS and EAE is a phenomenon known as epitope spreading (Tuohy and Kinkel, 2000). Epitope spreading is believed to occur when there is major tissue damage, and debris from the destroyed tissue is taken up, processed, and presented by immune cells, leading to inflammatory response toward new epitopes (Lehmann et al., 1993). During the course of the disease, T cells develop immunogenicity to new myelin proteins. This causes further destruction that leads to chronic tissue damage of the myelin sheath but, more importantly, it creates more difficulties in developing antigenspecific therapies. Epitope spreading can occur intramolecularly, in which case some mice develop autoreactivity toward $\mathrm{MBP}_{35-47}, \mathrm{MBP}_{81-100}$, and $\mathrm{MBP}_{121-140}$ over time when $\mathrm{MBP}_{1-11}$ is the initiating antigen (Lehmann et al., 1992). In addition, there is evidence of intermolecular epitope spreading where, during the course of the disease, proliferative responses toward PLP are present in MBP-induced mice (Perry et al., 1991). There are no reports in the literature of epitope spreading between $\mathrm{MOG}_{38-50}$ and $\mathrm{PLP}_{139-151}$, but we believe that the efficacy of PLP-BPI in the MOG-induced model and the additive efficacy of $\mathrm{MVB}_{\mathrm{MOG} / \mathrm{PLP}}$ are possibly due to epitope spreading. We propose that MVB $\mathrm{BOG}_{\mathrm{PLP}}$ suppressed the T-cell response for MOG as well as PLP. This strategy of multi-antigenic immunosuppression has been employed previously. Multi-antigenic peptide therapies made of up four different antigens $\left(\mathrm{PLP}_{139-151}, \mathrm{PLP}_{178-191}, \mathrm{MBP}_{84-104}\right.$, and $\left.\mathrm{MOG}_{92-106}\right)$ fixed to splenic APC have been developed and have shown efficacy in suppressing EAE induced by all four antigens (Smith and Miller, 2006).

BPI molecules are made up of two main components covalently linked to each other (Manikwar et al., 2011). The first component is the antigenic peptide and the second is the adhesion peptide. It is proposed that the antigenic peptide portion binds to the MHC-II molecule on the surface of APC, and the adhesion peptide, which is derived from the LFA-1 protein, binds to ICAM-1, also on the surface of APC. Traditionally, we used a linker composed of aminocaproic acid and glycine to link both peptide components in BPI molecules. For the synthesis of MOG-BPI and MVB $\mathrm{MOG}_{\text {PLP, }}$ a polyethylene glycol-3 $\left(\mathrm{PEG}_{3}\right)$ linker was used to improve solubility. Previously, we saw no significant difference in the use of $\mathrm{PEG}_{3}$ or aminocaproic acid as a linker. We propose that, due to the presence of the covalent linker, the BPI molecule tethers the loaded MHC-II molecule and ICAM-1, thus hindering the formation of the immunological synapse. This prevents the initiation of an inflammatory response to the specific antigen recognized by the MHC-II molecule. With the MVB, the idea has been expanded to incorporate more than one antigenic peptide. It is proposed that each antigenic peptide will be recognized by its respective MHC-II molecule and will suppress the inflammatory response to that antigen.

MS and EAE are characterized by severe inflammation with high levels of IL-6 (Maimone et al., 1997) and IFN- $\gamma$ (Kebir et al., 2009) found in the CNS. Moreover, their role in the pathogenesis of disease is significant. In addition to recruitment of other inflammatory immune cells, IL-6 is a mediator in increasing the permeability of the BBB (Abbott et al., 2006, Prendergast and Anderton, 2009), and IFN- $\gamma$ has been reported to prevent remyelination of the CNS (Lin et al., 2006). Therefore, in order to treat or suppress the disease, therapies aimed at reducing the inflammatory response are vital. Currently on the market, glatiramer acetate, a drug used for the treatment of MS, works by diverting the immune response away from the $\mathrm{T}_{\mathrm{H}} 1$ phenotype (Neuhaus et al., 2001, Dhib-Jalbut, 2003). 
The cytokine data from the present study demonstrated that when MOG-BPI and $\mathrm{MVB}_{\mathrm{MOG} / \mathrm{PLP}}$ were administered to mice with MOG-induced EAE, the inflammatory cytokines IL- 6 and IFN- $\gamma$ were suppressed significantly by both peptides, thus shifting the balance away from the inflammatory response. In addition, the proliferation assay from

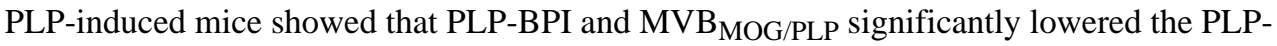
responsive population of splenocytes.

PLP-BPI molecules have been shown to suppress the production of IL-17 in PLP-stimulated EAE, implying that BPI molecules suppress the $\mathrm{T}_{\mathrm{H}} 17$ cell proliferation (Ridwan et al., 2010, Badawi et al., 2012). In contrast, PLP-BPI stimulates the production of IL-10 (Ridwan et al., 2010). The reduction of IL-6 has been shown to suppress the differentiation of $T_{H} 17$ and $\mathrm{T}_{\mathrm{H}} 1$ phenotypes to protect against EAE development (Serada et al., 2008). PLP-BPI also protects the CNS by inhibiting the breakdown of the BBB as measured by leakiness of the BBB (Badawi et al., 2012). The leakiness was determined by measuring brain disposition of Gadolinium-DTPA (Gd-DTPA) as determined by magnetic resonance imaging (MRI) after its delivery in normal mice, diseased mice, and diseased mice treated with PLP-BPI. The non-treated EAE-mice had the highest brain disposition of Gd-DTPA compared to normal mice and PLP-BPI-treated EAE mice, while PLP-BPI-treated mice had Gd-DTPA brain disposition similar to that in normal mice. This indicates that PLP-BPI can prevent the BBB breakdown while suppressing EAE. In another study, a BPI molecule called PLP-cIBR suppressed the demyelination in the CNS of EAE mice compared to PBS-treated mice (Kiptoo et al., 2013).

Although $\mathrm{MVB}_{\mathrm{MOG} / \mathrm{PLP}}$ has not been used to reverse EAE after the disease has progressed, other PLP-BPI molecules have been shown to reverse the PLP-stimulated EAE disease. In our previous study, PLP-BPI molecules were injected with a maximum of 3 injections (100 nmol/injection) after the animals showed disease scores of one or worse. The results showed that BPI treatments produced faster remission of the disease than PBS treatment (Kobayashi et al., 2008, Ridwan et al., 2010). In the future, a similar study will be carried out to compare the efficacy of $\mathrm{MVB}_{\mathrm{MOG} / \mathrm{PLP}}$ with that of other BPI molecules in reversing the disease after it progresses; in addition, BPI molecules will be evaluated for preventing disease relapse in the PLP-stimulated EAE model. Finally, the efficacy and mechanisms of action of $\mathrm{MVB}_{\mathrm{MOG} / \mathrm{PLP}}$ as a therapeutic vaccine will also be evaluated.

In conclusion, developing molecules that can target more than one epitope is critical for making BPI technology more applicable for MS. In human disease, the identity of the inciting antigen is not usually known. In addition, since the disease is frequently not diagnosed early, other antigens of the myelin sheath become targeted by immune cells due to epitope spreading. Therefore, molecules that can target many antigens are critically important for the treatment of MS or EAE irrespective of the inciting antigens or antigenic spread. Thus far, we have developed a peptide that is composed of two antigenic peptides. For future studies, we would like to expand this peptide to incorporate other immunodominant antigens to suppress disease initiated by other antigens as well as to avoid the problem of intra- and inter-molecular epitope spreading. 


\section{Acknowledgments}

We thank the National Institutes of Health (R01-AI-063002 and R56-AI-063002) and National Multiple Sclerosis Society for supporting this work. Ahmed H. Badawi was also supported by the Multidimensional Vaccinogenesis Training Grant (NIAID, T32-NIH-066335). We are grateful for the help of Nancy Harmony in proofreading the manuscript.

\section{References}

Abbott NJ, Ronnback L, Hansson E. Astrocyte-endothelial interactions at the blood-brain barrier. Nature reviews Neuroscience. 2006; 7:41-53.

Badawi AH, Kiptoo P, Wang WT, Choi IY, Lee P, Vines CM, et al. Suppression of EAE and prevention of blood-brain barrier breakdown after vaccination with novel bifunctional peptide inhibitor. Neuropharmacology. 2012; 62:1874-81. [PubMed: 22210333]

Bielekova B, Muraro PA, Golestaneh L, Pascal J, McFarland HF, Martin R. Preferential expansion of autoreactive T lymphocytes from the memory T-cell pool by IL-7. Journal of neuroimmunology. 1999; 100:115-23. [PubMed: 10695722]

Dhib-Jalbut S. Glatiramer acetate (Copaxone) therapy for multiple sclerosis. Pharmacol Ther. 2003; 98:245-55. [PubMed: 12725872]

Goebels N, Hofstetter H, Schmidt S, Brunner C, Wekerle H, Hohlfeld R. Repertoire dynamics of autoreactive $\mathrm{T}$ cells in multiple sclerosis patients and healthy subjects: epitope spreading versus clonal persistence. Brain: a journal of neurology 123 Pt. 2000; 3:508-18.

Grakoui A, Bromley SK, Sumen C, Davis MM, Shaw AS, Allen PM, et al. The immunological synapse: a molecular machine controlling T cell activation. Science. 1999; 285:221-7. [PubMed: 10398592]

Kebir H, Ifergan I, Alvarez JI, Bernard M, Poirier J, Arbour N, et al. Preferential recruitment of interferon-gamma-expressing TH17 cells in multiple sclerosis. Ann Neurol. 2009; 66:390-402. [PubMed: 19810097]

Kiptoo P, Buyuktimkin B, Badawi AH, Stewart J, Ridwan R, Siahaan TJ. Controlling immune response and demyelination using highly potent bifunctional peptide inhibitors in the suppression of experimental autoimmune encephalomyelitis. Clin Exp Immunol. 2013; 172:23-36. [PubMed: 23480182]

Kobayashi N, Kiptoo P, Kobayashi H, Ridwan R, Brocke S, Siahaan TJ. Prophylactic and therapeutic suppression of experimental autoimmune encephalomyelitis by a novel bifunctional peptide inhibitor. Clinical immunology. 2008; 129:69-79. [PubMed: 18676182]

Kobayashi N, Kobayashi H, Gu L, Malefyt T, Siahaan TJ. Antigen-specific suppression of experimental autoimmune encephalomyelitis by a novel bifunctional peptide inhibitor. The Journal of pharmacology and experimental therapeutics. 2007; 322:879-86. [PubMed: 17522343]

Lassmann H. Classification of demyelinating diseases at the interface between etiology and pathogenesis. Current opinion in neurology. 2001; 14:253-8. [PubMed: 11371746]

Lee KH, Holdorf AD, Dustin ML, Chan AC, Allen PM, Shaw AS. T cell receptor signaling precedes immunological synapse formation. Science. 2002; 295:1539-42. [PubMed: 11859198]

Lehmann PV, Forsthuber T, Miller A, Sercarz EE. Spreading of T-cell autoimmunity to cryptic determinants of an autoantigen. Nature. 1992; 358:155-7. [PubMed: 1377368]

Lehmann PV, Sercarz EE, Forsthuber T, Dayan CM, Gammon G. Determinant spreading and the dynamics of the autoimmune T-cell repertoire. Immunology today. 1993; 14:203-8. [PubMed: 7686009]

Lin W, Kemper A, Dupree JL, Harding HP, Ron D, Popko B. Interferon-gamma inhibits central nervous system remyelination through a process modulated by endoplasmic reticulum stress. Brain: a journal of neurology. 2006; 129:1306-18. [PubMed: 16504972]

Maimone D, Guazzi GC, Annunziata P. IL-6 detection in multiple sclerosis brain. J Neurol Sci. 1997; 146:59-65. [PubMed: 9077497] 
Manikwar P, Kiptoo P, Badawi AH, Buyuktimkin B, Siahaan TJ. Antigen-specific blocking of CD4specific immunological synapse formation using BPI and current therapies for autoimmune diseases. Medicinal research reviews. 2011

Muraro PA, Wandinger KP, Bielekova B, Gran B, Marques A, Utz U, et al. Molecular tracking of antigen-specific $\mathrm{T}$ cell clones in neurological immune-mediated disorders. Brain: a journal of neurology. 2003; 126:20-31. [PubMed: 12477694]

Murray JS, Oney S, Page JE, Kratochvil-Stava A, Hu Y, Makagiansar IT, et al. Suppression of type 1 diabetes in NOD mice by bifunctional peptide inhibitor: modulation of the immunological synapse formation. Chemical biology \& drug design. 2007; 70:227-36. [PubMed: 17718717]

Neuhaus O, Farina C, Wekerle H, Hohlfeld R. Mechanisms of action of glatiramer acetate in multiple sclerosis. Neurology. 2001; 56:702-8. [PubMed: 11288751]

Perry LL, Barzaga-Gilbert E, Trotter JL. T cell sensitization to proteolipid protein in myelin basic protein-induced relapsing experimental allergic encephalomyelitis. Journal of neuroimmunology. 1991; 33:7-15. [PubMed: 1711539]

Prendergast CT, Anderton SM. Immune cell entry to central nervous system--current understanding and prospective therapeutic targets. Endocrine, metabolic \& immune disorders drug targets. 2009; 9:315-27.

Ridwan R, Kiptoo P, Kobayashi N, Weir S, Hughes M, Williams T, et al. Antigen-specific suppression of experimental autoimmune encephalomyelitis by a novel bifunctional peptide inhibitor: structure optimization and pharmacokinetics. The Journal of pharmacology and experimental therapeutics. 2010; 332:1136-45. [PubMed: 20026673]

Serada S, Fujimoto M, Mihara M, Koike N, Ohsugi Y, Nomura S, et al. IL-6 blockade inhibits the induction of myelin antigen-specific Th17 cells and Th1 cells in experimental autoimmune encephalomyelitis. Proc Natl Acad Sci U S A. 2008; 105:9041-6. [PubMed: 18577591]

Smith CE, Miller SD. Multi-peptide coupled-cell tolerance ameliorates ongoing relapsing EAE associated with multiple pathogenic autoreactivities. J Autoimmun. 2006; 27:218-31. [PubMed: 17289470]

Tseng SY, Dustin ML. T-cell activation: a multidimensional signaling network. Current opinion in cell biology. 2002; 14:575-80. [PubMed: 12231352]

Tuohy VK, Kinkel RP. Epitope spreading: a mechanism for progression of autoimmune disease. Arch Immunol Ther Exp (Warsz). 2000; 48:347-51. [PubMed: 11140461]

Tuohy VK, Yu M, Weinstock-Guttman B, Kinkel RP. Diversity and plasticity of self recognition during the development of multiple sclerosis. J Clin Invest. 1997; 99:1682-90. [PubMed: 9120012]

Tuohy VK, Yu M, Yin L, Kawczak JA, Kinkel PR. Regression and spreading of self-recognition during the development of autoimmune demyelinating disease. J Autoimmun. 1999a; 13:11-20. [PubMed: 10441163]

Tuohy VK, Yu M, Yin L, Kawczak JA, Kinkel RP. Spontaneous regression of primary autoreactivity during chronic progression of experimental autoimmune encephalomyelitis and multiple sclerosis. The Journal of experimental medicine. 1999b; 189:1033-42. [PubMed: 10190894]

van der Merwe PA. Formation and function of the immunological synapse. Current opinion in immunology. 2002; 14:293-8. [PubMed: 11973125]

Vanderlugt CJ, Miller SD. Epitope spreading. Current opinion in immunology. 1996; 8:831-6. [PubMed: 8994863]

Voskuhl RR, Martin R, Bergman C, Dalal M, Ruddle NH, McFarland HF. T helper 1 (Th1) functional phenotype of human myelin basic protein-specific T lymphocytes. Autoimmunity. 1993; 15:13743. [PubMed: 7692995]

Youssef S, Stuve O, Patarroyo JC, Ruiz PJ, Radosevich JL, Hur EM, et al. The HMG-CoA reductase inhibitor, atorvastatin, promotes a Th2 bias and reverses paralysis in central nervous system autoimmune disease. Nature. 2002; 420:78-84. [PubMed: 12422218]

Zhang J, Markovic-Plese S, Lacet B, Raus J, Weiner HL, Hafler DA. Increased frequency of interleukin 2-responsive $\mathrm{T}$ cells specific for myelin basic protein and proteolipid protein in peripheral blood and cerebrospinal fluid of patients with multiple sclerosis. The Journal of experimental medicine. 1994; 179:973-84. [PubMed: 7509366] 
Zhao H, Kiptoo P, Williams TD, Siahaan TJ, Topp EM. Immune response to controlled release of immunomodulating peptides in a murine experimental autoimmune encephalomyelitis (EAE) model. Journal of controlled release: official journal of the Controlled Release Society. 2010; 141:145-52. [PubMed: 19748537]

\section{Abbreviations used}

EAE experimental autoimmune encephalomyelitis

BPI bifunctional peptide inhibitor

PLP proteolipid protein

MOG myelin oligodendrocyte glycoprotein

MVB multivalent BPI 


\section{Highlights}

1. A novel peptide composed of MOG and an adhesion peptide was tested in EAE.

2. Another peptide composed of 2 myelin antigens and an adhesion peptide was tested.

3. MOG-BPI suppressed disease significantly when tested in MOG-induced EAE.

4. $\mathrm{MVP}_{\mathrm{MOG} / \mathrm{PLP}}$ was able to suppress both MOG- and PLP-induced EAE. 


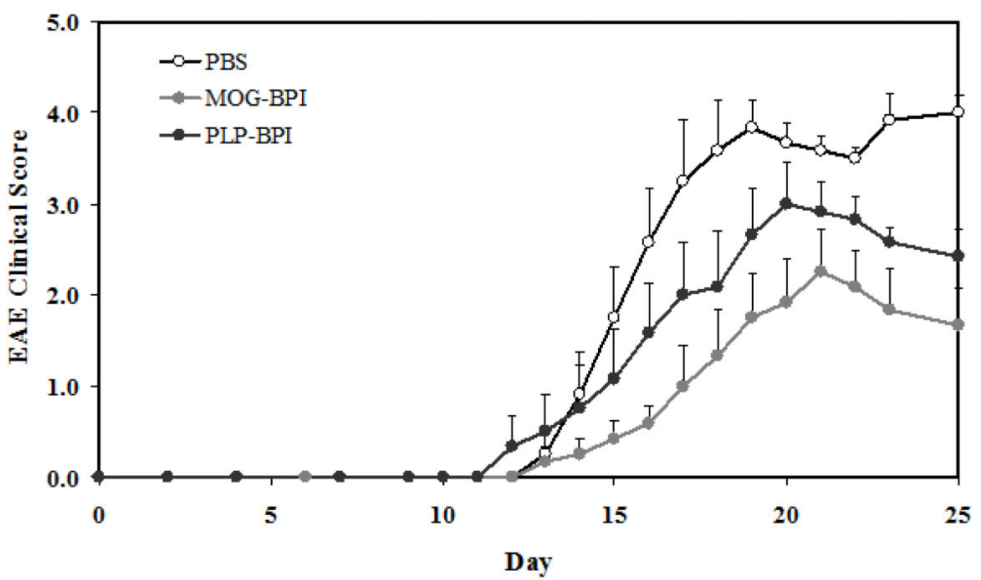

Fig. 1A

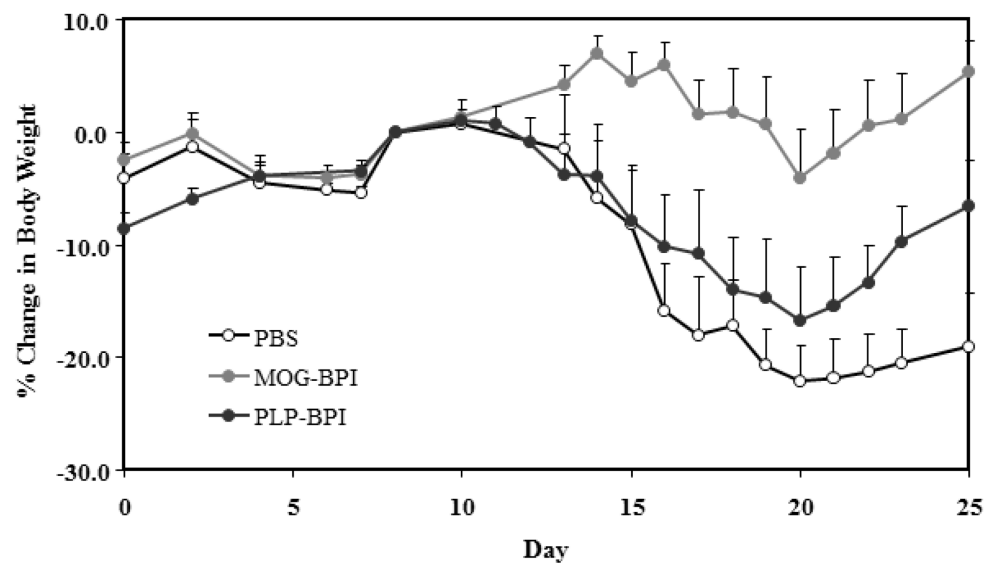

Fig. 1B

J Neuroimmunol. Author manuscript; available in PMC 2014 October 15. 


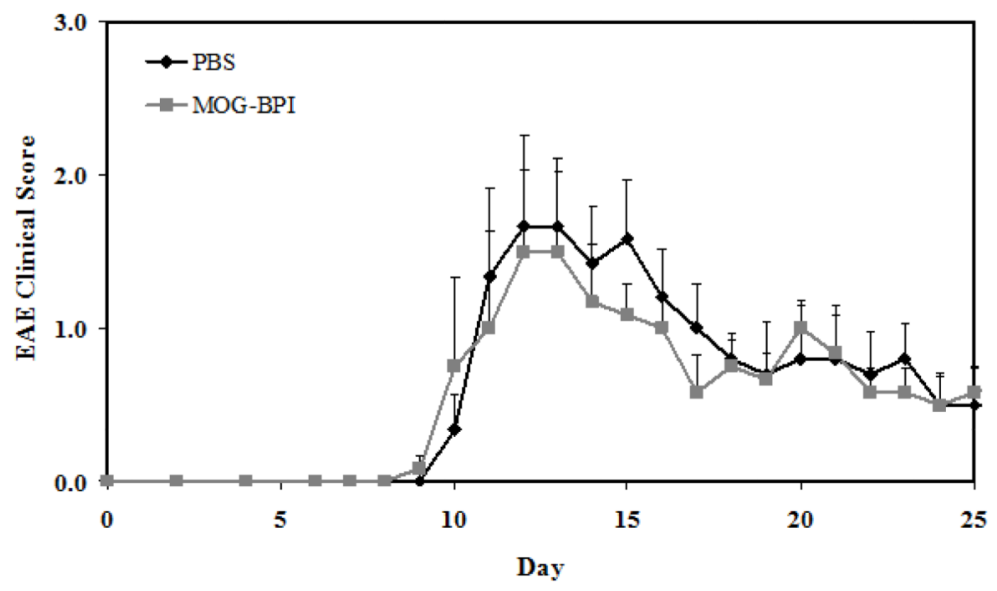

Fig. 1C

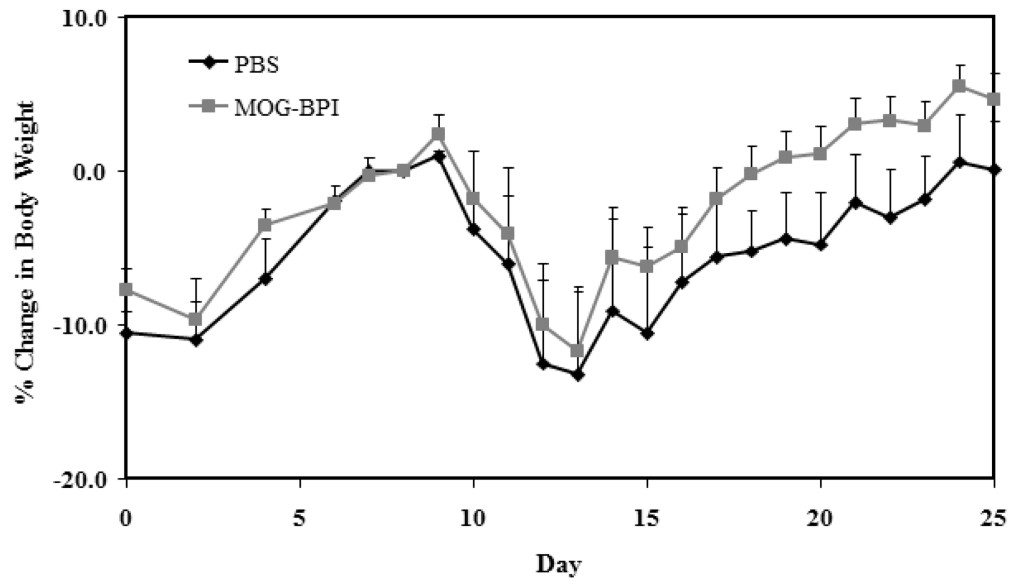

Fig. 1D

Figure 1.

In vivo cross-reactivity of MOG-BPI and PLP-BPI. PBS (100 $\mu \mathrm{l})$ and peptides (100 nmol/100 $\mu \mathrm{l}$ ) were administered s.c. on days 4, 7, and 10. MOG-BPI- and PLP-BPI-treated mice were compared to PBS-treated mice in the MOG-induced EAE model and efficacy was determined by (A) clinical disease score of EAE, expressed as the mean clinical score \pm SEM $(n=6)$, and (B) percent change in body weight, expressed as the mean $\%$ change in body weight \pm SEM $(n=6)$. The efficacy of MOG-BPI in suppressing disease in PLPinduced EAE mice was determined using the $(\mathrm{C})$ clinical disease score of EAE, expressed as 
the mean clinical score $\pm \operatorname{SEM}(n=6)$, and (D) percent change in body weight, expressed as the mean $\%$ change in body weight $\pm \operatorname{SEM}(n=6)$. 


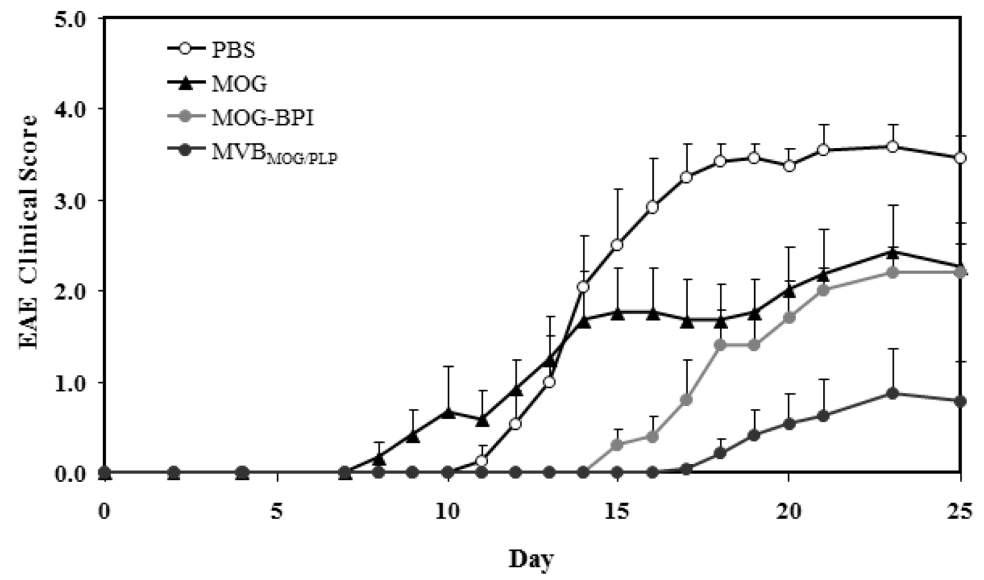

Fig. 2A

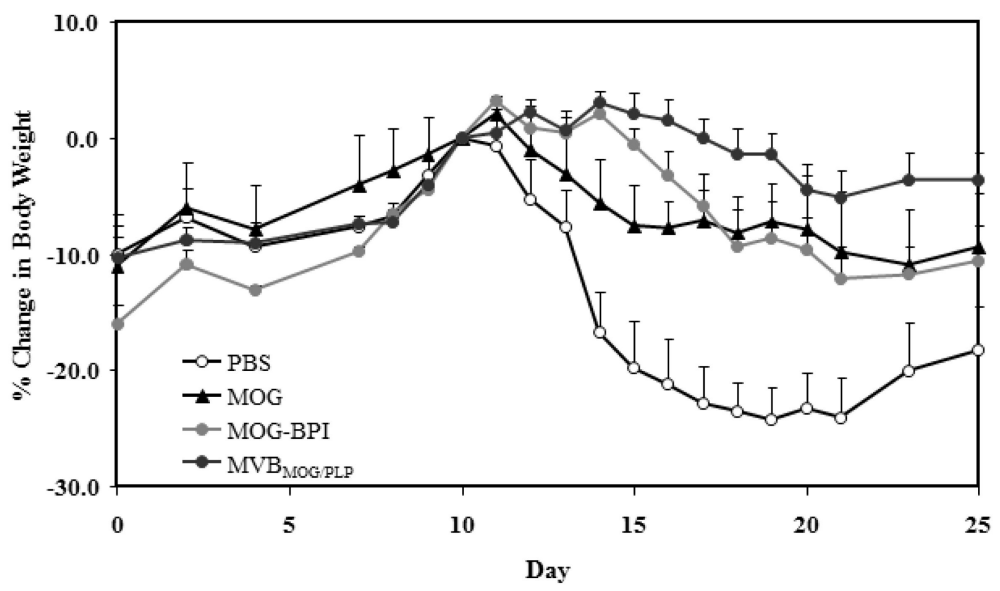

\section{Fig. 2B}

Figure 2.

In vivo efficacies of $\mathrm{MVB}_{\mathrm{MOG} / \mathrm{PLP}}$ and controls in suppressing MOG-induced EAE. PBStreated mice $(n=12)$ received s.c. injections of $100 \mu \mathrm{PBS}$ on days 4,7 , and 10 . MOG- $(n=$ $6)$, MOG-BPI- $(n=5)$, and MVB MOG/PLP- $(n=12)$ treated mice received $100 \mathrm{nmol} / 100 \mu \mathrm{l}$ PBS on days 4, 7, and 10 (s.c). The efficacy of each peptide was determined by (A) clinical disease score of EAE, expressed as the mean clinical score \pm SEM, and (B) percent change in body weight, expressed as the mean $\%$ change in body weight \pm SEM. 


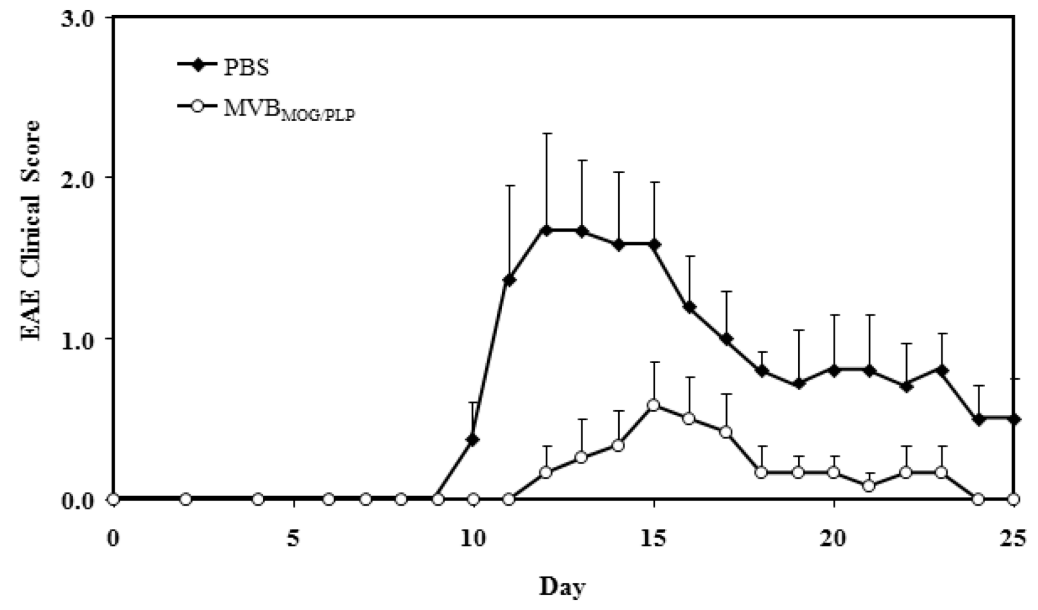

Fig. 3A

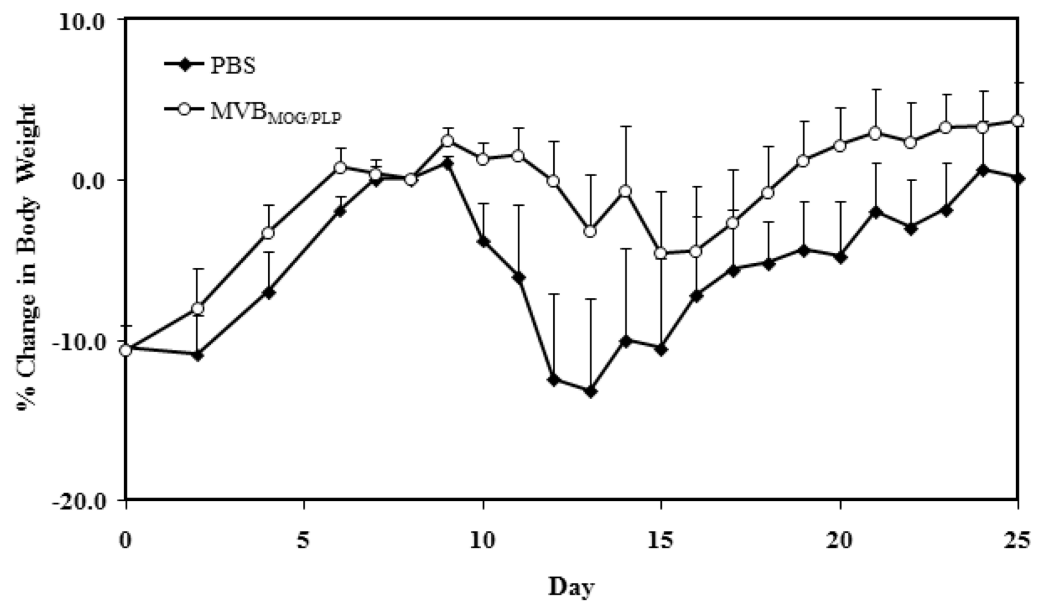

Fig. 3B

Figure 3.

In vivo efficacy of $\mathrm{MVB}_{\mathrm{MOG} / \mathrm{PLP}}$ in suppressing PLP-induced $\mathrm{EAE}$. MVB $\mathrm{MOG}_{\mathrm{PLP}}$-treated mice $(n=6)$ received three s.c. injections at a concentration of $100 \mathrm{nmol} / 100 \mu \mathrm{l}$ on days 4 , 7 , and 10 and its efficacy was compared to that of PBS in mice $(n=6)$ treated in a similar fashion. Progress of the disease was monitored following (A) the clinical disease score, expressed as the mean clinical score \pm SEM, and (B) the percent change in body weight, expressed as the mean $\%$ change in body weight \pm SEM. 


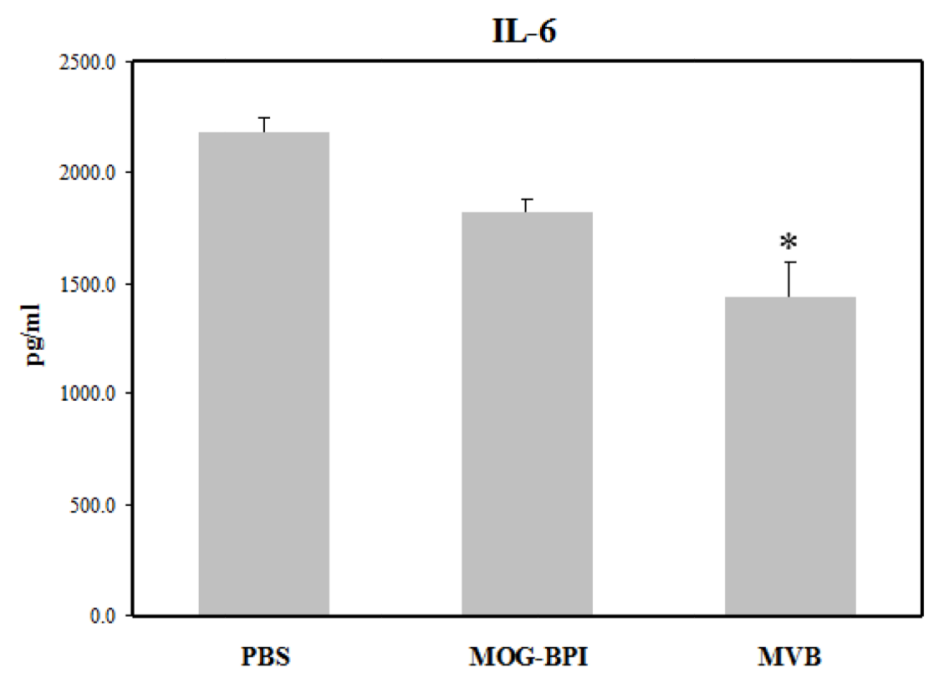

Fig. 4A

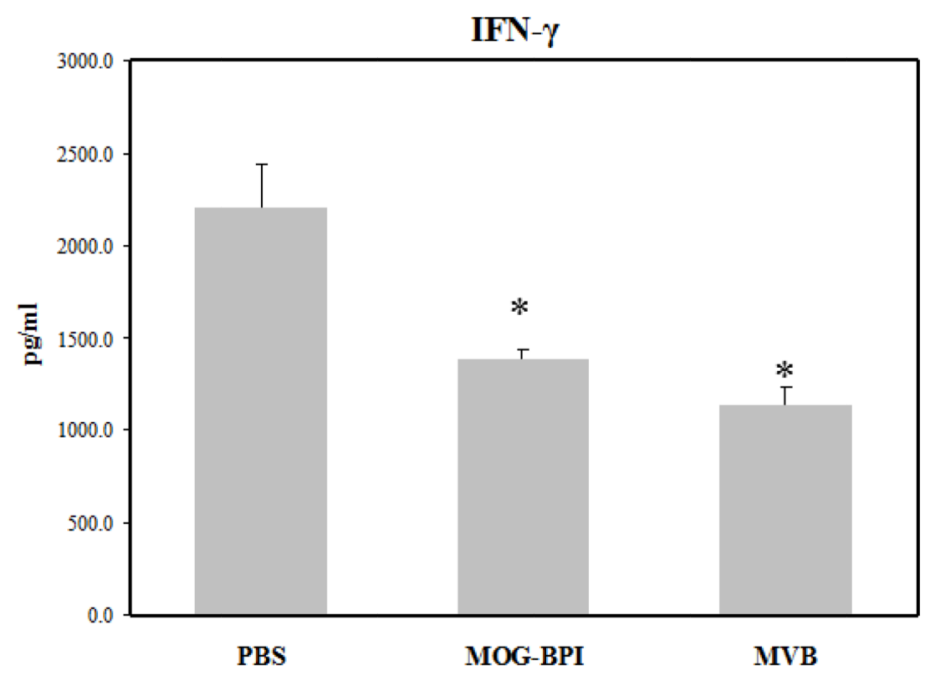

Fig. 4B

Figure 4.

Concentrations of pro-inflammatory cytokines (A) IL-6 and (B) IFN- $\gamma$ from the cell culture supernatant. Splenocytes were isolated from the spleens of MOG-induced EAE mice on day 30. The three different groups consisted of PBS-, MOG-BPI-, or MVB MOG/PLP- treated mice on days 4,7 , and 10 . The pooled splenocytes $(n=3$ mice) were stimulated in vitro with $\mathrm{PLP}_{139-151}$, and supernatant was isolated 72 hours later for cytokine detection $(* p<0.01)$. 


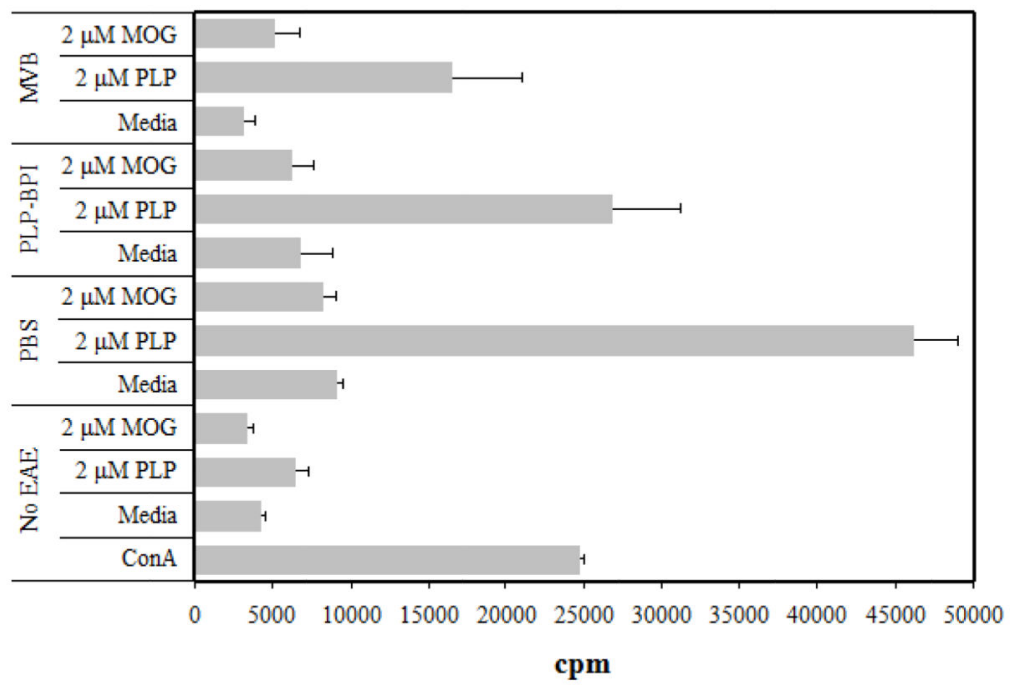

Fig. 5

Figure 5.

Incorporation of $\left[{ }^{3} \mathrm{H}\right]$ thymidine in splenocytes isolated from PLP-induced EAE mice on day 30. EAE-free mice were stimulated with ConA, medium, PLP $(2 \mu \mathrm{M})$, or MOG $(2 \mu \mathrm{M})$ and the remaining treated groups were stimulated with only medium, PLP $(2 \mu \mathrm{M})$, and MOG (2 $\mu \mathrm{M})$. The three treatment groups consisted of PBS-, PLP-BPI-, or MVB $\mathrm{MOG}_{\text {PLP- }}$ treated mice on days 4,7 , and 10 . Each group $(n=3)$ was pulsed with $\left[{ }^{3} \mathrm{H}\right]$ thymidine 72 hours after stimulation and incorporated radioactivity was measured the following day. 


\section{Table 1}

\section{List of peptides used in the present study}

Acp in the linker represents $\varepsilon$-aminocaproic acid. Ac- represents the acetyl-capped $\mathrm{N}$-terminus of the peptide. $\mathrm{NH}_{2}$ represents the amide-capped C-terminus of the peptide. $\mathrm{X}$ represents polyethyleneglycol-3.

\begin{tabular}{ll}
\hline Peptide & Sequence \\
\hline PLP $_{139-151}$ (PLP) & HSLGKWLGHPDKF \\
MOG $_{38-50}$ (MOG) & GWYRSPFSRVVHL \\
PLP-BPI & Ac-HSLGKWLGHPDKF-(AcpGAcpGAcp) ${ }_{2}$-ITDGEATDSG-NH ${ }_{2}$ \\
MOG-BPI & Ac-GWYRSPFSRVVHL-XGX-ITDGEATDSG-NH ${ }_{2}$ \\
MVB $_{\text {MOG/PLP }}$ & Ac-GWYRSPFSRVVHL-XGX-ITDGEATDSG-XGX-HSLGKWLGHPDKF-NH \\
\hline
\end{tabular}


Table 2

Summary of in vivo studies

\begin{tabular}{lccc}
\hline Group $^{a}$ & Induction antigen & Incidence of disease $\boldsymbol{b}^{\boldsymbol{1}}$ & Mean maximal score \pm SEM \\
\hline In vivo Study I & & & \\
PBS & MOG & $6 / 6$ & $4.00 \pm 0.18$ \\
PLP-BPI & MOG & $6 / 6$ & $3.00 \pm 0.47$ \\
MOG-BPI & MOG & $6 / 6$ & $2.25 \pm 0.46$ \\
PBS & PLP & $6 / 6$ & $1.67 \pm 0.59$ \\
MOG-BPI & PLP & $6 / 6$ & $1.50 \pm 0.53$ \\
In vivo Study II & & & \\
PBS & MOG & $12 / 12$ & $3.58 \pm 0.24$ \\
MOG & MOG & $5 / 6$ & $2.42 \pm 0.52$ \\
MOG-BPI & MOG & $5 / 5$ & $2.20 \pm 0.31$ \\
MVB & MOG & $4 / 12$ & $0.88 \pm 0.49$ \\
In vivo Study III & & & \\
PBS & PLP & $6 / 6$ & $1.67 \pm 0.59$ \\
MVB & PLP & $3 / 6$ & $0.58 \pm 0.27$ \\
\hline
\end{tabular}

\footnotetext{
${ }^{a}$ All injections were administered s.c. at $100 \mathrm{nmol}(100 \mu \mathrm{lPS})$ on days 4, 7, and 10.

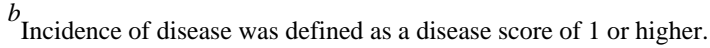

